\title{
Health education and the control of intestinal worm infections in China: a new vision
}

\author{
Donald P McManus ${ }^{1 *}$, Franziska A Bieri ${ }^{1,2,3}$, Yue-Sheng Li ${ }^{1,2,4}$, Gail M Williams², Li-Ping Yuan ${ }^{4}$, Yang Henglin ${ }^{5}$, \\ Zun-Wei Du ${ }^{5}$, Archie CA Clements ${ }^{3}$, Peter Steinmann ${ }^{6}$, Giovanna Raso ${ }^{6}$, Peiling Yap ${ }^{6}$, Ricardo J Soares Magalhães ${ }^{7}$, \\ Donald Stewart ${ }^{8}$, Allen G Ross ${ }^{8}$, Kate Halton ${ }^{9}$, Xiao-Nong Zhou ${ }^{10}$, Remigio M Olveda ${ }^{11}$, Veronica Tallo ${ }^{11}$ \\ and Darren J Gray ${ }^{1,2,3}$
}

\begin{abstract}
Background: The transmission of soil-transmitted helminths (STHs) is associated with poverty, poor hygiene behaviour, lack of clean water and inadequate waste disposal and sanitation. Periodic administration of benzimidazole drugs is the mainstay for global STH control but it does not prevent re-infection, and is unlikely to interrupt transmission as a stand-alone intervention.

Findings: We reported recently on the development and successful testing in Hunan province, PR China, of a health education package to prevent STH infections in Han Chinese primary school students. We have recently commenced a new trial of the package in the ethnically diverse Xishuangbanna autonomous prefecture in Yunnan province and the approach is also being tested in West Africa, with further expansion into the Philippines in 2015.

Conclusions: The work in China illustrates well the direct impact that health education can have in improving knowledge and awareness, and in changing hygiene behaviour. Further, it can provide insight into the public health outcomes of a multi-component integrated control program, where health education prevents re-infection and periodic drug treatment reduces prevalence and morbidity.
\end{abstract}

Keywords: Ascaris lumbricoides, Trichuris trichiura, Necator americanus, Ancylostoma duodenale, Soil-transmitted helminths (STHs), People's Republic of China, Health education, "Magic Glasses" video

\section{Findings}

Roundworms (Ascaris lumbricoides), whipworms (Trichuris trichiura) and hookworms (Necator americanus and Ancylostoma duodenale) are intestinal parasitic nematodes that are the scourge of many developing countries due to the fact that chronically infected individuals can succumb to a variety of clinical complications, including poor physical and mental development [1,2]. Transmission of these soiltransmitted helminths (STHs) is associated with poverty, poor hygiene behaviour, lack of clean water and inadequate waste disposal and sanitation [3]. Consequently, high rates of infection are common in deprived urban as well as in rural areas, where people have limited or no access to health care and preventive measures [1]. Globally,

\footnotetext{
* Correspondence: Don.McManus@qimrberghofer.edu.au

${ }^{1}$ QIMR Berghofer Medical Research Institute, Herston, Brisbane, Queensland, Australia

Full list of author information is available at the end of the article
}

an estimated 438.9 million people were infected with hookworm in 2010, 819.0 million with A. lumbricoides and 464.6 million with $T$. trichiura [4].

In the People's Republic of China (PR China), the 2001-2004 second national survey of parasitic diseases indicated there were 129 million STH infections with the highest prevalence occurring in children aged 5 to 14 years, emphasising the continued importance of STHs as a significant public health problem in the country [5]. To overcome the challenge posed by STHs and other parasitic infections, the Chinese Ministry of Health announced in 2005 the "National Control Program on Important Parasitic Diseases from 2006 to 2015" which set the target of reducing parasite prevalence by $70 \%$ by the year 2015 [5].

Currently the key strategy for global STH control in endemic areas is the periodic administration of anthelminthic drugs, e.g. albendazole or mebendazole, to high 
risk groups, especially school-aged children [6]. While this strategy is effective in achieving morbidity control, it does not prevent re-infection, which usually occurs rapidly, and therefore is not likely to interrupt transmission as a stand-alone intervention [7-10]. Additional preventive public health measures, such as improvements in hygiene behaviour achieved through health education, are required to achieve the sustainable control of these parasitic worms.

We reported recently on the development and testing of a health education package that included a 12-minute animated narrative cartoon video entitled "The Magic Glasses" (accessible at: http://www.nejm.org/action/showMediaPlayer?doi=10.1056\%2FNEJMoa1204885\&aid =NEJMoa1204885_attach_1\&area) to prevent STH infections in Chinese primary school students. The cluster randomized controlled trial, conducted in Linxiang City District, Hunan province, and involving 1718 children aged 9-10 years in 38 rural schools over the course of 1 school year (September 2010 through June 2011), resulted in the health education package having a $50 \%$ efficacy in preventing STH infections [11]. The trial established the proof of principle that health education can increase knowledge and change behavior, resulting in fewer intestinal worm infections. Crucial to the success of this study was the very early involvement in the study of health workers, health and education officials, teachers, parents and students, and a thorough appraisal of their knowledge, attitudes, and practices regarding STHs and the risk factors associated with infection. Consideration of all these features enabled the development of a culturally tailored, informative, and engaging educational package; but to show broader application, our findings required further validation in another epidemiological and cultural setting in China.

In 2013 we commenced a new trial of the educational package in two counties of the ethnically diverse Xishuangbanna autonomous prefecture in Yunnan province, where the force of STH infection is higher $(>50 \%$ prevalence) [12] than Hunan province (10\% prevalence). Xishuangbanna is located in the south of the province and borders the Lao People's Democratic Republic and Myanmar. The prefecture has approximately 1 million inhabitants, of whom approximately $30 \%$ are Han Chinese, $30 \%$ are Dai and the remainder belong to a diverse group of other ethnic minorities. The study design is similar to that of the trial we undertook in Hunan province. Involving 9-10 year old schoolchildren, the cluster-randomised controlled trial will determine the impact of the videobased health education package on knowledge, hygiene behaviour, and STH infection outcomes. Forty schools, each with at least 38 grade 4 children, have been randomly selected in two counties, Jinghong and Menghai, both predominantly non-Han, using a spatial sampling frame to minimise contamination. Of these, 20 schools have been randomly assigned intervention status and 20 as controls. Each student in grade 4 has been recruited into the study cohort and will be followed over 2 school years. Primary endpoints will be incidence and intensity of STH infection and knowledge about the worms, their transmission, symptoms, treatment and prevention; secondary endpoints will be change in observed hand washing behaviour; tertiary endpoints will be measures of morbidity (haemoglobin levels to assess anaemia, height and weight to reflect growth and malnutrition) and educational outcomes (school academic performance and attendance). We will also evaluate the cost-effectiveness of the video-based health education package both on its own and in combination with anthelminthic chemotherapy. The costs will be compared to chemotherapy alone, helping to guide STH control policy in Yunnan, other parts of China and beyond. To date, there have been few economic evaluations of parasite control programs and, generally, these cost and cost-effectiveness analyses have evaluated treatment components only [13].

The research we are undertaking in China is designed to establish an evidence-base for a health educational package for use in schools that can be readily incorporated into the school curriculum in the framework of, for example, a regional de-worming program. The package effectively complements the current approach to STH control advocated by the World Health Organisation [6] and can, following adaptation to local cultural realities, be deployed in non-Chinese settings as well. The approach is currently being tested in Côte d'Ivoire in West Africa and we will further expand into the Philippines in 2015. Future programs could also involve the integration of chemotherapy and health education with WASH (water, sanitation and hygiene) efforts to ensure access to clean water and good sanitation in addition to improved personal hygiene [14-16]. Ultimately, the work in China has the potential to be a model for other neglected tropical diseases (NTDs) because it illustrates the direct impact that health education can have in improving knowledge and awareness, and in changing hygiene behaviour, thereby reducing the chances of an individual becoming infected. Further, it can provide insight into the public health outcomes of a multi-component integrated control program, where health education prevents reinfection and periodic drug treatment is used to reduce prevalence and morbidity.

\section{Conclusion}

Having already successfully pioneered multi-component integrated strategies, which included health education, for the control of other NTDs such as lymphatic filariasis and schistosomiasis [17-20], the Chinese authorities are in an ideal position to undertake a multi-component 
integrated public health strategy that combines mass drug administration (MDA) with health education and improved WASH for the control of STH in rural China. Based on the national-scale experience, China would then be in a good position to help implement such projects in other countries.

\section{Competing interests}

The authors declare that they have no competing interests.

\section{Authors' contributions}

DPM conceived the article and DPM and FB produced the first draft. All other authors read the draft and commented on the content. All authors read and approved the final manuscript.

\section{Acknowledgements}

The "Magic Glasses" health education program in China and the Philippines is supported by UBS Optimus Foundation, Zurich, Switzerland and the National Health and Medical Research Council (NHMRC) of Australia. We thank Andrew Bedford, director of the cartoon "The Magic Glasses," and the team at 5th World Media that produced it. Dr. Gray is an Australian Research Council Fellow (DECRA) and Dr. McManus is a NHMRC Senior Principal Research Fellow.

\section{Author details}

'QIMR Berghofer Medical Research Institute, Herston, Brisbane, Queensland, Australia. ${ }^{2}$ The School of Population Health, The University of Queensland, Brisbane, Queensland, Australia. ${ }^{3}$ Research School of Population Health, The Australian National University, Canberra, Australia. ${ }^{4}$ Hunan Institute of Parasitic Diseases, WHO Collaborating Centre for Research and Control on Schistosomiasis in Lake Region, Yueyang, China. ${ }^{5}$ Yunnan Institute of Parasitic Diseases, Pu'er, China. ${ }^{6}$ Swiss Tropical and Public Health Institute, University of Basel, Basel, Switzerland. ' 5 School of Veterinary Science, University of Queensland, Brisbane, Gatton, Australia. ${ }^{8}$ Griffith Health Institute, Griffith University, Brisbane, Australia. ${ }^{9}$ School of Public Health and Social Work, Queensland University of Technology, Brisbane, Australia. ${ }^{10}$ National Institute of Parasitic Disease, Chinese Center for Disease Control and Prevention, Shanghai, China. ${ }^{1}$ Research Institute for Tropical Medicine, Manila, Philippines.

Received: 9 July 2014 Accepted: 21 July 2014

Published: 24 July 2014

\section{References}

1. Bethony J, Brooker S, Albonico M, Geiger SM, Loukas A, Diemert D, Hotez PJ: Soil-transmitted helminth infections: ascariasis, trichuriasis, and hookworm. Lancet 2006, 367:1521-1532.

2. Yap P, Du Z-W, Chen R, Zhang L-P, Wu F-W, Wang J, Wang X-Z, Zhou H, Zhou X-N, Utzinger J, Steinmann P: Soil-transmitted helminth infections and physical fitness in school-aged Bulang children in southwest China: results from a cross-sectional survey. Parasit Vectors 2012, 5:50

3. Nasr N, Al-Mekhlafi H, Ahmed A, Roslan MA, Bulgiba A: Towards an effective control programme of soil-transmitted helminth infections among Orang Asli in rural Malaysia. Part 2: Knowledge, attitude, and practices. Parasit Vectors 2013, 6:28.

4. Pullan R, Smith J, Jasrasaria R, Brooker S: Global numbers of infection and disease burden of soil transmitted helminth infections in 2010 . Parasit Vectors 2014, 7:37.

5. Chen Y-D, Tang L-H, Xu L-Q: Current status of soil-transmitted nematode infection in China. Biomed Environ Sci 2008, 21:173-179.

6. World Health Organization: Accelerating Work to Overcome the Global Impact of Neglected Tropical Diseases - A Roadmap for Implementation. GenevaSwitzerland: World Health Organization; 2012.

7. Truscott1 JE, Hollingsworth TD, Brooker SJ, Anderson RM: Can chemotherapy alone eliminate the transmission of soil transmitted helminths? Parasit Vectors 2014, 7:266.

8. Gunawardena K, Kumarendran B, Ebenezer R, Gunasingha MS, Pathmeswaran A, de Silva N: Soil-transmitted helminth infections among plantation sector schoolchildren in Sri Lanka: prevalence after ten years of preventive chemotherapy. PLoS Neg/ Trop Dis 2011, 5:e1341.
9. Hotez P: Hookworm and poverty. Ann N Y Acad Sci 2008, 1136:38-44.

10. Jia TW, Melville S, Utzinger J, King CH, Zhou XN: Soil-transmitted helminth reinfection after drug treatment: a systematic review and meta-analysis. PLoS Negl Trop Dis 2012, 6:e1621.

11. Bieri FA, Gray DJ, Williams GM, Raso G, Li Y-S, Yuan L, He Y, Li RS, Guo F-Y, Li Y-S, McManus DP: Health-education package to prevent worm infections in Chinese schoolchildren. N Engl J Med 2013, 368:1603-1612.

12. Mofid LS, Bickle Q, Jiang JY, Du ZW, Patrick E: Soil-transmitted helminthiasis in rural south-west China: prevalence, intensity and risk factor analysis. Southeast Asian J Trop Med Public Health 2011, 42:513-526.

13. Conteh L, Engels T, Molyneux D: Socioeconomic aspects of neglected tropical diseases. Lancet 2010, 375:239-247.

14. Bieri F, Yuan L-P, Li Y-S, He Y-K, Bedford A, Li R, Guo F-Y, Li S-M, Williams G, McManus D, Raso G, Gray DJ: Development of an educational cartoon to prevent worm infections in Chinese schoolchildren. Infect Dis Poverty 2013, 2:29

15. Bieri FA, Li Y-S, Yuan L-P, He Y-K, Gray DJ, Williams GM, McManus DP: School- based health education targeting intestinal worms - further support for integrated control. PLoS Negl Trop Dis 2014, 8:e2621.

16. Pose RR: Neglected Tropical Diseases: the Case of Cambodia. London, UK: Development Progress: Overseas Development Institute; 2014:1-7.

17. Molyneux DH: Elimination of transmission of lymphatic filariasis in Egypt. Lancet 2006, 367:966-968.

18. Hotez PJ, Molyneux DH, Fenwick A, Kumaresan J, Sachs SE, Sachs JD, Savioli L: Control of neglected tropical diseases. N Engl J Med 2007, 357:1018-1027.

19. Utzinger J, Zhou XN, Chen MG, Bergquist R: Conquering schistosomiasis in China: the long march. Acta Trop 2005, 96:69-96.

20. Yang GJ, Liu L, Zhu HR, Griffiths SM, Tanner M, Bergquist R, Utzinger J, Zhou $\mathrm{XN}$ : China's sustained drive to eliminate neglected tropical diseases. Lancet Infect Dis 2014, 23. S1473-3099 (14)70727-3. doi:10.1016/S1473-3099 (14)70727-3. [Epub ahead of print].

\section{doi:10.1186/1756-3305-7-344}

Cite this article as: McManus et al:: Health education and the control of intestinal worm infections in China: a new vision. Parasites \& Vectors 2014 7:344.

\section{Submit your next manuscript to BioMed Central and take full advantage of:}

- Convenient online submission

- Thorough peer review

- No space constraints or color figure charges

- Immediate publication on acceptance

- Inclusion in PubMed, CAS, Scopus and Google Scholar

- Research which is freely available for redistribution 